\title{
NOTES
}

\section{Radical Copolymerization Reactivity of 1-Butadienyloxytrimethylsilane}

\author{
Hiroshi Sumi, Takumi Noto, Yoshihito InaI, \\ Tadamichi HirabaYashi, and Kenji YoKota \\ Department of Materials Science and Engineering, \\ Nagoya Institute of Technology, \\ Gokiso-cho, Showa-ku, Nagoya 466, Japan \\ (Received January 14, 1994)
}

\begin{abstract}
KEY WORDS Radical Copolymerization / 1-Butadienyloxytrimethylsilane
/ Styrene / Methyl Methacrylate / Monomer Reactivity Ratio / $Q-e$ Value /
\end{abstract}

We have been interested in the aldol condensation-type of group transfer polymerization of 1-butadienyloxytrimethylsilane (BdTMS). ${ }^{1}$

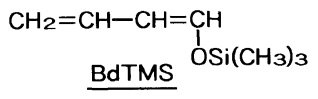

BdTMS is a silyl ether and conjugated diene monomer. It seems to be an attractive subject how BdTMS behaves in conventional polymerization methods. If BdTMS can be polymerized through conventional procedures, this monomer would be useful for molecular design of functional polymers because of the diene monomer with hydroxyl group masked by trimethylsilyl group. However, there is little data concerning the polymerization of BdTMS, in contrast with much application of BdTMS in the field of organic synthesis. ${ }^{2}$

Thus, the present paper deals with radical copolymerization between BdTMS and styrene (St) as well as methyl methacrylate (MMA) to clarify the fundamental character of BdTMS as a monomer.

\section{EXPERIMENTAL}

\section{Materials}

BdTMS was prepared according to Danishe- fsky's procedure. ${ }^{3}$ The details are given in our previous papers. ${ }^{1}$ BdTMS containing more than $99 \% E$-isomer was obtained by fractional distillation and redistilled on calcium hydride under reduced pressure before use. St and MMA were purified by the conventional method, and distilled before use. Azobisisobutyronitrile (AIBN) was purified by recrystallization from methanol.

\section{Copolymerization}

A dry glass tube was charged with AIBN, BdTMS, and St under nitrogen atmosphere. The contents were frozen and the tube was sealed in vacuo. Polymerization was carried out at $60^{\circ} \mathrm{C}$. After an adequate period, the contents were poured into a large amount of methanol containing a small amount of hydroquinone. The copolymer was reprecipitated by pouring benzene solution into methanol, and dried in vacuo at room temperature until constant weight was achieved. The freeze-drying technique was adopted in certain series of experiments. Polymerization of BdTMS with MMA was carried out at $70^{\circ} \mathrm{C}$ using AIBN as an initiator. 
Analyses

${ }^{1} \mathrm{H}$ and ${ }^{13} \mathrm{C}$ NMR spectra were recorded with a Varian XL-200 spectrometer. Molecular weight was determined by a gel permeation chromatography (GPC), calibrating molecular weight with standard polystyrenes. GPC was measured by differential refractometry in tetrahydrofuran as the eluent on a Tosoh HLC-803D instrument equipped with four gel packed columns (TSKgel, G5000-, G4000-, G3000-, G2000-HXL).

\section{RESULTS AND DISCUSSION}

Radical Copolymerization of BdTMS-St and BdTMS-MMA

Radical copolymerization of BdTMS with St was carried out at various compositions of monomer feed. Polymerization conditions and

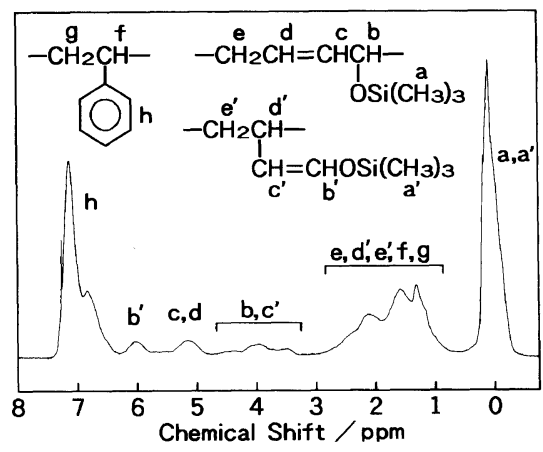

Figure 1. ${ }^{1} \mathrm{H}$ NMR spectrum of poly(BdTMS-co-St): $39.1 \mathrm{~mol} \%$ of BdTMS. results are summarized in Table I. As BdTMS content in the monomer feed increased, it took longer time to obtain polymer owing to poor homopolymerizability of BdTMS. Figure 1 shows the ${ }^{1} \mathrm{H}$ NMR spectrum of the typical poly(BdTMS-co-St). The 1,4 and 3,4 structures of BdTMS unit existed in the copolymer. The 1,2 structure was not observed. The assignment of each signal is shown in Figure 1. Copolymer compositions were determined from ${ }^{1} \mathrm{H}$ NMR spectroscopy by comparing the intensity of resonance peaks due to total trimethylsilyl (a and $\mathrm{a}^{\prime}$ in Figure 1) and phenyl (h in Figure 1) protons. The contents of 1,4 and 3,4 structures of BdTMS unit are shown in Table I. The content of the 3,4 structure of BdTMS unit in radical copolymerization was considerably large in comparison with that in group transfer polymerization. Figure 2 shows a composition

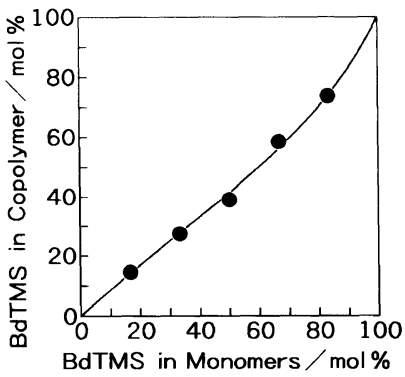

Figure 2. Copolymer composition of BdTMS in relation to monomer composition for polymerization of BdTMS with St: - experimental point; curve, calculated from the reactivity ratios $r_{1}=0.500$ and $r_{2}=1.130$

Table I. Copolymerization of BdTMS $\left(M_{1}\right)$ with ST $\left(M_{2}\right)$ at $60^{\circ} \mathrm{C}$

\begin{tabular}{|c|c|c|c|c|c|c|c|c|c|}
\hline \multirow{2}{*}{ No. } & \multicolumn{3}{|c|}{ Monomer feed } & \multirow{2}{*}{$\frac{\mathrm{AIBN}}{\mathrm{mmol}}$} & \multirow{2}{*}{$\frac{\text { Time }}{\mathrm{h}}$} & \multirow{2}{*}{$\frac{\text { Yield }}{\%}$} & \multicolumn{3}{|c|}{ Polymer } \\
\hline & $\mathrm{M}_{1} / \mathrm{mmol}$ & $\mathbf{M}_{2} / \mathrm{mmol}$ & $\begin{array}{l}\mathrm{mol}^{\%} \\
\text { of } \mathrm{M}_{1}\end{array}$ & & & & $\begin{array}{l}\mathrm{mol} \% \\
\text { of } \mathrm{M}_{1}\end{array}$ & $\frac{M_{n}{ }^{\mathrm{a}}}{\times 10^{3}}$ & $1,4: 3,4^{b}$ \\
\hline 1 & 9.64 & 50.45 & 16.7 & 0.61 & 1.2 & 2.0 & 14.6 & 11.8 & $49: 51$ \\
\hline 2 & 19.33 & 40.32 & 33.3 & 0.61 & 2.5 & 1.3 & 27.4 & 9.4 & $48: 52$ \\
\hline 3 & 28.97 & 30.29 & 50.0 & 0.61 & 16 & 2.7 & 39.1 & 7.5 & $44: 56$ \\
\hline 4 & 38.66 & 20.16 & 66.7 & 0.61 & 36 & 6.2 & 58.4 & 3.6 & $44: 56$ \\
\hline 5 & 48.30 & 10.12 & 83.3 & 0.61 & 50 & 4.0 & 73.8 & 3.4 & $41: 59$ \\
\hline
\end{tabular}

a Calibrated by standard polystyrene samples.

b Microstructure contents of BdTMS determined by ${ }^{1} \mathrm{H}$ NMR. 
Table II. Copolymerization of BdTMS $\left(\mathrm{M}_{1}\right)$ with MMA $\left(\mathrm{M}_{2}\right)$ at $70^{\circ} \mathrm{C}$

\begin{tabular}{|c|c|c|c|c|c|c|c|c|c|}
\hline \multirow{3}{*}{ No. } & \multicolumn{3}{|c|}{ Monomer feed } & \multirow{3}{*}{$\frac{\mathrm{AIBN}}{\mathrm{mmol}}$} & \multirow{3}{*}{$\frac{\text { Time }}{\mathrm{h}}$} & \multirow{3}{*}{$\frac{\text { Yield }}{\%}$} & \multicolumn{3}{|c|}{ Polymer } \\
\hline & \multirow[b]{2}{*}{$\mathrm{M}_{1} / \mathrm{mmol}$} & \multirow[b]{2}{*}{$\mathrm{M}_{2} / \mathrm{mmol}$} & \multirow[b]{2}{*}{$\begin{array}{l}\mathrm{mol}^{\%} \\
\text { of } \mathrm{M}_{1}\end{array}$} & & & & \multirow[b]{2}{*}{$\begin{array}{l}\mathrm{mol} \% \\
\text { of } \mathrm{M}_{1}\end{array}$} & \multirow{2}{*}{$\frac{M_{n}^{\mathrm{a}}}{\times 10^{3}}$} & \multirow[b]{2}{*}{$1,4: 3,4^{b}$} \\
\hline & & & & & & & & & \\
\hline 1 & 11.40 & 56.09 & 16.9 & 0.68 & 2 & 3.7 & 33.6 & 7.0 & $59: 41$ \\
\hline 2 & 22.80 & 46.74 & 32.8 & 0.70 & 5 & 7.3 & 44.8 & 4.8 & $57: 43$ \\
\hline 3 & 28.50 & 28.05 & 50.4 & 0.57 & 20 & 7.7 & 50.1 & 7.5 & $50: 50$ \\
\hline 4 & 34.20 & 18.70 & 64.7 & 0.53 & 20 & 7.1 & 55.0 & 4.7 & $47: 53$ \\
\hline 5 & 45.60 & 9.35 & 83.0 & 0.55 & 51 & 5.4 & 63.1 & 8.6 & $53: 47$ \\
\hline
\end{tabular}

a Calibrated by standard polystyrene samples.

b Microstructure contents of BdTMS determined by ${ }^{1} \mathrm{H}$ NMR.

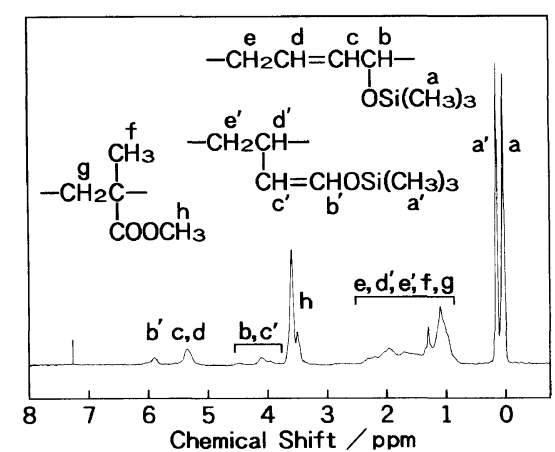

Figure 3. ${ }^{1} \mathrm{H}$ NMR spectrum of poly(BdTMS-coMMA): $44.8 \mathrm{~mol} \%$ of BdTMS.

diagram of copolymer against monomer feed in copolymerization of BdTMS-St.

Similarly, radical copolymerization of BdTMS with MMA was carried out. Conditions and results are summarized in Table II. Figure 3 shows the ${ }^{1} \mathrm{H}$ NMR spectrum of the typical poly(BdTMS-co-MMA). Copolymer compositions were determined from ${ }^{1} \mathrm{H}$ NMR method by comparing the intensity of the resonance peaks due to total trimethylsilyl (a and $\mathrm{a}^{\prime}$ in Figure 3) and methoxy (h in Figure 3 ) protons. Figure 4 shows a composition diagram of copolymer against monomer feed in copolymerization of BdTMS-MMA. Using MMA as a comonomer led to increase in the tendency of the alternating arrangement of BdTMS and MMA units in the copolymer.

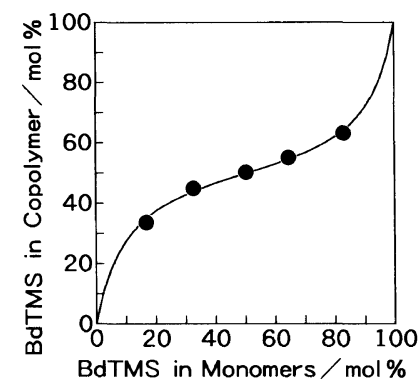

Figure 4. Copolymer composition of BdTMS in relation to monomer composition for polymerization of BdTMS with MMA: experimental point; curve, calculated from the reactivity ratios $r_{1}=0.175$ and $r_{2}=0.189$.

\section{Monomer Reactivity Ratios, $Q$ and e Values of $B d T M S$}

Monomer reactivity ratios in the above copolymerizations were evaluated by KelenTüdös method ${ }^{4}$ as $r_{1}=0.500$ and $r_{2}=1.130$ for $\operatorname{BdTMS}\left(\mathrm{M}_{1}\right)-\mathrm{St}\left(\mathrm{M}_{2}\right)$, while $r_{1}=0.175$ and $r_{2}=0.189$ for $\operatorname{BdTMS}\left(\mathrm{M}_{1}\right)-\operatorname{MMA}\left(\mathrm{M}_{2}\right)$. The $Q-e$ scheme has been proposed by Alfrey and Price $^{5}$ for systematizing much copolymerization data. The values of BdTMS are calculated to be $Q=1.62$ and $e=-1.56$ when comonomer is $\mathrm{St}$, and to be $Q=1.89$ and $e=-1.45$ when comonomer is MMA.

$Q$ and $e$ values of some conjugated diene and vinyl ether monomers are listed in Table III to compare with those of BdTMS. The $Q$ value of BdTMS is obviously close to that of typical conjugated diene monomers rather than that 
Table III. $Q-e$ values, $\lambda_{\max }$, and ${ }^{13} \mathrm{C}$ chemical shifts of various diene and vinyl ether monomers

\begin{tabular}{|c|c|c|c|c|}
\hline \multirow{2}{*}{ Monomer } & \multirow{2}{*}{$Q^{\mathbf{a}}$} & \multirow{2}{*}{$e^{\mathbf{a}}$} & \multirow{2}{*}{$\frac{\lambda_{\max }^{\mathrm{b}}}{\mathrm{nm}}$} & \multirow{2}{*}{$\frac{\text { Chemical shift }^{\mathrm{c}}}{\mathrm{ppm}}$} \\
\hline & & & & \\
\hline $\operatorname{BdTMS}(\mathrm{ST})$ & 1.62 & -1.56 & 232.5 & 80.8 \\
\hline (MMA) & 1.89 & -1.45 & & \\
\hline Butadiene & 2.39 & -1.05 & 217 & 75.3 \\
\hline Isoprene & 3.33 & -1.22 & 220 & 76.4 \\
\hline 2,3-Dimethyl-1,3-butadiene & 5.86 & -1.81 & 226 & 79.8 \\
\hline 2-Chloro-1,3-butadiene & 7.26 & -0.02 & 223 & \\
\hline Hexachloro-1,3-butadiene & 1.31 & 0.76 & 220 & 69.2 \\
\hline Ethyl vinyl ether & 0.032 & -1.17 & & 108.5 \\
\hline Butyl vinyl ether & 0.087 & -1.20 & 192 & 108.1 \\
\hline Isobutyl vinyl ether & 0.023 & -1.77 & & 108.7 \\
\hline
\end{tabular}

a Data in ref 6 except for BdTMS.

b Data in ref 7 and 8 except for BdTMS.

c ${ }^{13} \mathrm{C}$ chemical shifts from $\mathrm{CS}_{2}$ : 4-position carbon for dienes, 2-position carbon for vinyl ethers.

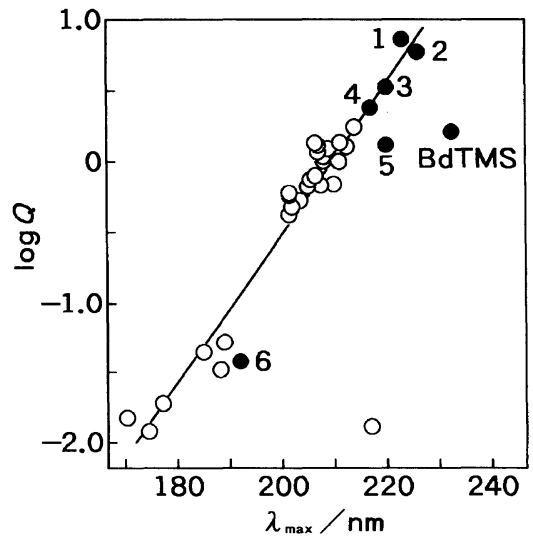

Figure 5. Plots of $\lambda_{\max }$ against $\log Q:$ 1) 2-chloro-1,3butadiene; 2) 2,3-dimethyl-1,3-butadiene; 3) isoprene; 4) butadiene; 5) hexachloro-1,3-butadiene; 6) butyl vinyl ether; $\bigcirc$, ref 7 .

of vinyl ether monomers. Table III lists wavelength $\left(\lambda_{\max }\right)$ at the maximum absorbance due to $\pi \rightarrow \pi^{*}$ transition observed in UV spectrum. Ito et al. ${ }^{7}$ reported that a linear correlation of $Q$ values against $\lambda_{\max }$ is observed for many vinyl monomers. Therefore it must be valid to interpret $Q$ as a measure of resonance effect in monomers. Following Ito's manner, Figure 5 shows the plots of $\log Q$ against $\lambda_{\max }$ for various monomers together with a plot of BdTMS. Although BdTMS is

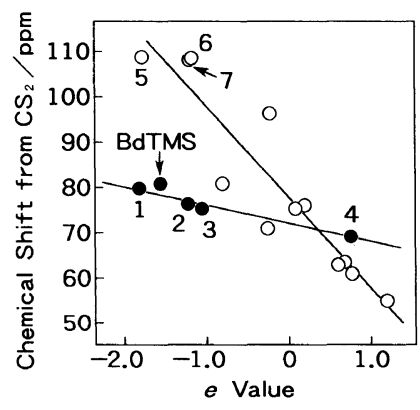

Figure 6. Plots of $e$ against ${ }^{13} \mathrm{C}$ chemical shift: 1) 2,3-dimethyl-1,3-butadiene; 2) isoprene; 3) butadiene; 4) hexachloro-1,3-butadiene; 5) isobutyl vinyl ether; 6) ethyl vinyl ether; 7) butyl vinyl ether; $O$, ref 9.

surely located in the region of conjugated dienes, it deviates somewhat from the normal correlation line toward longer wavelength. This bathochromic shift can be justified by taking into account the $\mathrm{p} \pi-\mathrm{d} \pi$ double bond character of the $\mathrm{O}-\mathrm{Si}$ bond. Such extension in the conjugated system may be sensitive to UV. The $Q$ value of BdTMS does not reflect resonance effect so much as that of conventional monomers, for copolymerization parameters are based on many participating factors.

$e$ of BdTMS is found to be more negative than those of conjugated diene monomers. This means that the electron-donating character of 
the trimethylsilyloxy group is stronger than that of other substituents. The electron density of the 2- or 4-position carbon of BdTMS should reflect the character. Hatada et al. ${ }^{9}$ pointed out that the chemical shifts of the 2-position carbon ( $\beta$-carbon) of a series of vinyl monomers are well correlated to $e .{ }^{13} \mathrm{C}$ chemical shifts of the 4-position carbon for conjugated dienes and the 2-position carbon for vinyl ethers are listed in Table III. Figure 6 shows plots of ${ }^{13} \mathrm{C}$ chemical shifts against $e$ for conjugated diene and vinyl monomers together with a plot of BdTMS. In Figure 6, plots are divided into two groups, vinyl monomers and conjugated diene monomers. The chemical shift of the 4-position carbon for conjugated diene monomer shifted to a lower field as $e$ became larger. The more negative $e$ of BdTMS is due to the electrondonating character of the trimethylsilyloxy group.

\section{REFERENCES}

1. T. Hirabayashi, T. Ito, and K. Yokota, Polym. J., 20, 1041 (1988); T. Hirabayashi, T. Kawasaki, and K. Yokota, ibid., 22, 287 (1990); H. Sumi, T. Hirabayashi, Y. Inai, and K. Yokota, ibid., 24, 669 (1992).

2. P. Brownbridge, Synthesis, 85 (1983).

3. S. Danishefsky and T. Kitahara, J. Org. Chem., 40, 538 (1975).

4. T. Kelen and K. Tüdös, J. Macromol. Sci., Chem., A9, 1 (1975).

5. T. Alfrey, Jr. and C. C. Price, J. Polym. Sci., 2, 101 (1947).

6. J. Brandrup and E. H. Immergut, Ed., "Polymer Handbook," 2nd ed, Wiley-Interscience, New York, N. Y., 1975, p II-387.

7. T. Ito, T. Otsu, and M. Imoto, J. Polym. Sci., B, 4, 81 (1966).

8. J. G. Grasselli, Ed., "Atlas of Spectral Data and Physical Compounds," CRC Press, Cleveland, Ohio, 1973.

9. K. Hatada, K. Nagata, and H. Yuki, Bull. Chem. Soc. Jpn., 43, 3267 (1970). 